\title{
Impact of LTE Operating in Unlicensed Spectrum on Wi-Fi Using Real Equipment
}

\author{
Vasilis Maglogiannis, Dries Naudts, Pieter Willemen and Ingrid Moerman \\ Ghent University - iMinds, Department of Information Technology (INTEC) \\ Ghent, Belgium \\ \{vasilis.maglogiannis, dries.naudts, pieter.willemen, ingrid.moerman\}@intec.ugent.be
}

\begin{abstract}
The proliferation of mobile devices and the exponential growth of data transmitted over the air pushed the wireless community to find solutions in order to increase network capacity and fully exploit the available spectrum. Recently, 3GPP announced the operation of LTE in the unlicensed spectrum in order to offload the limited and expensive licensed spectrum. Concurrently, leading parties of the wireless community examine standalone operation of LTE in unlicensed spectrum. LTE was initially designed to operate in licensed spectrum and does not use any channel estimation mechanism to determine ongoing transmissions by other co-located networks. This introduces important coexistence challenges in unlicensed spectrum between LTE deployments and the current, well-established technologies, such as IEEE 802.11 (a.k.a. Wi-Fi). In this paper, we discuss the core differences between LTE and Wi-Fi, which lead to significant coexistence issues. We verify and showcase the problem by analyzing the performance degradation of $\mathrm{Wi}-\mathrm{Fi}$, when a traditional LTE network is co-located and operates in the same unlicensed frequency without any coexistence mechanism. The experiments are performed using open-source LTE and Wi-Fi implementations on real equipment in a fully controlled wireless environment. We conclude with showing the need for coexistence mechanisms, following the work that is being done within the standardization activities.
\end{abstract}

Keywords-LTE unlicensed; Wi-Fi; real equipment experimentation; coexistence; heterogeneous networks; performance

\section{INTRODUCTION}

Over the past few years, mobile devices such as smartphones, tablets, laptops and wearable technology have tremendously proliferated and changed the way people communicate, as they are online anytime and anywhere. According to Qualcomm, the already huge amount of traffic is expected to further increase by a factor of 1000 between 2010 and 2020 [1]. Hence, one can easily deduce that the wireless network capacity will soon become a bottleneck for this massive growth of wireless traffic.

LTE is a technology that approaches the Shannon limit and can assist significantly in solving the $1000 x$ challenge. LTE is a scheduled technology that uses innovative methods such as Hybrid Automatic Repeat Request (HARQ) and carrier aggregation. These techniques render LTE capable to manage the available spectrum more efficiently than its predecessors and to achieve high data rates, low latency, QoS guarantees and fairness. However, the amount of available licensed spectrum is expensive and becomes limited, as the wireless technologies that use it, such as LTE, are consolidated and are intensively used by a growing amount of users.

Attempting to overcome these drawbacks, key players in the mobile wireless community have submitted proposals to 3GPP, which would allow LTE to operate in unlicensed spectrum bands. Within 3GPP activities on the LTE operation in unlicensed spectrum (also known as LAA Licensed-Assisted Access) has been started, as an enhancement towards LTE Release 13 [2]. LTE LAA will give the operators the possibility to use a secondary cell operating in the unlicensed spectrum alongside the primary cell operating in the licensed band that they own. There are two predominant proposals for LTE operation in unlicensed spectrum. According to the first one, a secondary cell on unlicensed spectrum will be used for supplemental downlink (DL) traffic only, while the uplink (UL) traffic will be transmitted on the operator's licensed spectrum. In the second proposal both supplemental DL and UL traffic will be transmitted via the cell operating in unlicensed spectrum. Additionally, big industry names founded the LTE-U Forum [3], which publishes specifications for minimum performance and coexistence mechanisms for eNB and UE operating in unlicensed spectrum, closely following the 3GPP specifications.

In parallel, many leading parties of the mobile world are doing preliminary steps to establish the LTE-U operation in standalone mode. To this end, they formed the MulteFire Alliance [4]. Their objective is to let LTE operate solely in unlicensed spectrum, so that it can be deployed by Internet service providers, cable companies, mobile operators, enterprises, building owners, etc.

The introduction of LTE into the unlicensed spectrum can significantly assist in dealing with the exponential data growth and moreover, it can solve the capacity problem that mobile operators face in order to provide the desired user experience. Furthermore, LTE in the unlicensed spectrum could considerably help in the increasingly important offloading of cellular networks through direct communications [5]. Nonetheless, LTE is a technology that is initially designed to operate in the licensed spectrum, assuming exclusive use of the assigned spectrum. It does not make use of a Listen Before Talk (LBT) mechanism in order to sense the medium and avoid collision with other ongoing transmissions from co-located networks. Hence, introducing LTE in unlicensed spectrum as it is, may have a detrimental impact on other co-located technologies that operate in the same bands, such as Wi-Fi [6]. 
In this paper, we analyze in depth and on real hardware the intuitive observation that LTE dominates Wi-Fi in a shared spectrum access mode. We describe the preliminary designed transmission protocol used by each technology and we highlight the reasons that lead to harmful coexistence between LTE and Wi-Fi. Until today, the literature lacks of a study that showcases the coexistence issues using real equipment. Initially and similar to the most technological breakthroughs the concept was studied using mathematical analysis and simulations. This paper targets to close this gap and presents the experimental verification of the impact of LTE on Wi-Fi using open-source LTE and Wi-Fi implementations on real equipment in a fully controlled wireless environment. During the experimentation we adopt the standalone operation of LTE in the unlicensed spectrum. We introduce LTE in unlicensed spectrum as it was originally designed without taking into account any coexistence mechanism and we examine the impact of LTE on Wi-Fi in terms of throughput and round trip latency. The paper highlights the need for coexistence mechanisms and aims to be used as a springboard for contribution to the discussion in 3GPP standardization about LTE operation in unlicensed spectrum by proposing potential improvements.

\section{RELATED WORK}

Although the LTE operation in unlicensed spectrum has only been announced recently, the problem of coexistence between LTE and Wi-Fi has already attracted many researchers and key players in wireless community, who study and evaluate the WiFi performance degradation due to the presence of LTE. This performance evaluation is based mainly on mathematical models and simulations.

In [7] the authors investigate the deployment of LTE small cells instead of Wi-Fi by a mobile operator in a license-exempt band. Coexistence mechanisms with Wi-Fi are discussed, while UL performance analysis using simulation scenarios with both random and cluster placement is conducted. The results show that LTE can deliver significant capacity, even if it shares the spectrum with Wi-Fi networks

A study that evaluates the performance of LTE and Wi-Fi in a shared frequency band using a simulation scenario is presented in [8]. As shown, LTE has a negative impact on Wi-Fi, especially in the case where many Wi-Fi users try to access the network simultaneously. By introducing a muting technique to LTE, the performance of Wi-Fi was increased, while LTE was still able to retain a fairly good performance.

In a similar way, the authors in [9] evaluate through simulations the performance impact of LTE and Wi-Fi when both operate in the same frequency. They propose a coexistence mechanism that exploits blank LTE subframes in order to give opportunity to Wi-Fi to transmit. They conclude that topology, as well as the number and order of the blank subframes lead to different performance results.

A framework in which a femtocell can access both licensed and unlicensed spectrum is proposed in [10]. In order to enable coexistence between LTE and Wi-Fi the authors propose an algorithm that enhances LTE with a channel sensing capability. The proposed framework is modelled and verified via simulations and the results showed a total throughput improvement of both cellular and non-cellular users.

An analytical model for evaluating the performance of colocated LTE and Wi-Fi networks is developed and used to obtain baseline performance measures in [11]. The results of the model have been partially validated via experimental evaluation using USRP platforms. Moreover, the authors propose an internetwork coordination with logically centralized radio resource management across LTE and Wi-Fi towards a fair coexistence.

Until today and except for the validation of the proposed interference characterization models in [11], the work that has been done studying the impact of LTE in unlicensed spectrum on Wi-Fi is focused on simulations or mathematical modelling. Hence, the literature lacks a study that investigates the coexistence results using real LTE and Wi-Fi equipment. This paper bridges this gap and presents the performance evaluation of Wi-Fi, when it is co-located with LTE operating in unlicensed spectrum, in a fully controlled environment using open-source equipment for both LTE and Wi-Fi networks.

\section{LTE VS WI-FI}

Wi-Fi uses Orthogonal Frequency Division Multiplexing (OFDM) digital modulation scheme that divides the spectrum into multiple OFDM subcarriers spanning (a multiple of) $20 \mathrm{Mhz}$ bandwidth. DCF (Distributed Coordination Function) is the fundamental mechanism that $\mathrm{Wi}-\mathrm{Fi}$ uses to access the medium and is designed to be asynchronous and decentralized [12]. Moreover, Wi-Fi uses Carrier Sensing Multiple Access with Collision Avoidance (CSMA/CA) method to estimate the channel condition (idle or busy) every time a node has data to transmit. According to this contention-based protocol, a Wi-Fi node first has to listen to the shared medium to determine if another node is already transmitting. This procedure is known as Clear Channel Assessment (CCA).

CCA consists of two functions named Carrier Sense (CS) and Energy Detection (ED). The CS function refers to the ability of the receiver to listen to the medium, to detect and successfully decode an incoming Wi-Fi preamble. If this is the case and the detected signal power is higher or equal to $-82 \mathrm{dBm}$, then CCA reports the channel as busy for the timeslot that is indicated in the frame's Physical Layer Convergence Protocol (PLCP) header length field. On the other hand, if the incoming signal cannot be decoded, the ED is used. The ED function refers to the ability of the receiver to detect the energy level in the operating channel based on non-Wi-Fi signals that are sensed in the same frequency band introducing interference or corrupted Wi-Fi transmissions that cannot be decoded. If the energy level is higher or at least equal to $-62 \mathrm{dBm}$, then $\mathrm{CCA}$ reports the channel as busy. ED must sense the channel every time slot to estimate the energy level of the channel, as the length of time that the medium will be busy cannot be determined.

If the channel is busy the node has to postpone its transmission and wait for a free DCF Inter-Frame Space (DIFS), or if QoS is enabled, an Arbitration Inter-Frame Spacing (AIFS) period plus a random backoff time to avoid packet collisions. After a transmission, the node waits for an acknowledgement (ACK) during a Short Inter-Frame Space (SIFS) period. 
LTE uses Orthogonal Frequency Division Multiple Access (OFDMA) for the DL and Single-Carrier Frequency Division Multiple Access (SC-FDMA) for the UL, which are multi-user versions of the OFDM digital modulation scheme [13]. The available spectrum is divided into subcarriers and each subcarrier occupies $15 \mathrm{KHz}$ of bandwidth. The time domain is organized in timeslots of $0.5 \mathrm{~ms}$ duration. One timeslot corresponds to 7 OFDM symbols when Normal Cyclic prefix (CP) is used. Combining the subcarriers and timeslots, LTE defines the Resource Block (RB). The RB is a unit of transmission resource and consists of 1 slot in time domain and 12 subcarriers in frequency domain. An LTE radio frame has a duration of $10 \mathrm{~ms}$ and consists of 10 sub-frames, each of which lasts $1 \mathrm{~ms}$ corresponding to 2 slots, as can be seen in Fig. 1 .

LTE sends user traffic in the DL and in the UL using the Physical DL Shared Channel (PDSCH) and the Physical UL Shared Channel (PUSCH) respectively. In addition to user traffic, several resource blocks are allocated for control traffic such as synchronization signals, reference signals used by the UE to estimate the channel, HARQ ACK/NACK signals, etc.

LTE is a scheduled technology and does not require carrier sensing before transmission, as it has been designed to operate in licensed spectrum. In LTE, scheduling is performed by the LTE base station (eNB) on a sub-frame level as Fig. 2 depicts. This means that each $1 \mathrm{~ms}$ the assignment of the subframes to the active UEs can change.

By the protocols description above it becomes clear that when a Wi-Fi network is co-located with an LTE network operating in unlicensed spectrum in the way it was originally designed, significant coexistence issues arise. The scheduler of LTE will schedule transmissions regardless the presence of WiFi. This way it may directly interfere with potential Wi-Fi transmissions or act as hidden terminal. Especially in case of heavy loaded LTE network, it will monopolize the wireless resources resulting in the starvation of the Wi-Fi network.

\section{EQUIPMENT AND EXPERIMENTATION SETUP}

For the purposes of this study an LTE network with opensource equipment for eNodeB and UE [14] has been deployed and configured to operate in the unlicensed spectrum. Simultaneously, a Wi-Fi network operates in the same frequency band. The experiments were conducted on the LTE and Wi-Fi infrastructure of the $\mathrm{W}$-iLab2 testbed at iMinds [15].

The LTE network consists of 2 software-defined radio (SDR) EXPRESSMIMO2 (EXMIMO2) boards [16] that run the OpenAirInterface (OAI) software [17]. The attached radio daughter board covers a large part of the RF spectrum $(250 \mathrm{MHz}$ to $3.8 \mathrm{GHz}$ ) allowing the definition of channels in the unlicensed spectrum. On top of these boards the OAI software is running. OAI aims to provide an open-source solution for both the LTE

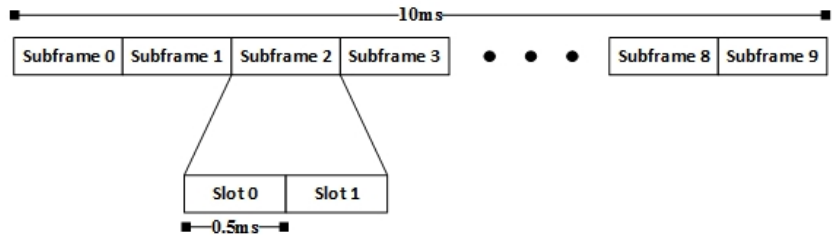

Fig. 1. An LTE frame structure that consists of 10 subframes, each of which has $1 \mathrm{~ms}$ duration. A subframe is divided into two slots of $0.5 \mathrm{~ms}$ duration.

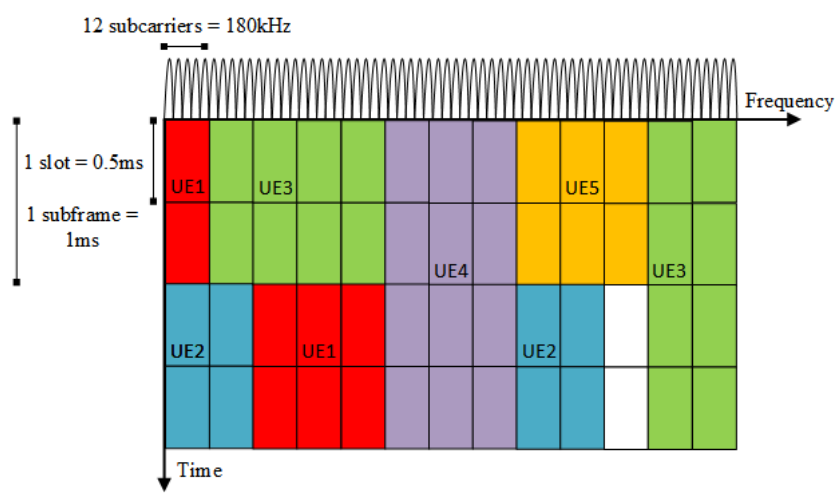

Fig. 2. LTE user traffic scheduling in frequency and time domain.

Evolved Packet Core (EPC) network and the LTE accessnetwork (EUTRAN) of 3GPP cellular systems. In our setup, one EXMIMO2 board has been configured to operate as eNB and the other as UE.

In order to enable LTE operation in unlicensed spectrum, a new band was defined in the OAI software, which uses the same center frequency as Wi-Fi channel $6(2.437 \mathrm{GHz})$. The width of the band is $5 \mathrm{MHz}$, as currently OAI permits LTE operation only in a $5 \mathrm{MHz}$ bandwidth. OAI supports both Frequency Division Duplex (FDD) and Time Division Duplex (TDD). In this study we focus on TDD mode, in order to investigate the interaction between Wi-Fi and LTE operating in a single frequency band for both DL and UL traffic. 3GPP defines 7 different DL/UL configuration profiles for the LTE TDD mode. TABLE I presents the 7 different TDD configurations, where " $D$ " and " $U$ " symbolize a DL and an UL subframe respectively, and "S" symbolizes a special subframe.

There are 9 different configurations for special subframe as can be seen in TABLE II. Each special subframe is divided into three parts: DwPTS (DL Pilot Time Slot), GP (Guard Period) and UpPTS (UL Pilot Time Slot). Different configurations allocate different number of OFDM symbols in each part. The GP is a transition gap between the DL and the UL. DwPTS is considered as a normal DL subframe and carries reference signals and control information, such as PSS (Primary Synchronization Signal). It can also carry data, when a configuration with a sufficient amount of OFDM symbols is selected. The UpPTS is primarily used for SRS (Sounding Reference Signals) transmission from the UE.

For the purpose of this study, the system has been configured to use TDD configuration profile " 3 ", providing a good proportion between the DL and UL timeslots in an LTE frame, and configuration " 0 " for the special subframe.

The Wi-Fi network consists of 2 nodes configured in infrastructure mode. One node operates as Access Point (AP) and the other as station (STA). Both the AP and the station use a Qualcomm Atheros AR928X wireless network adapter and the ath9k driver [18]. The Wi-Fi network operates in channel 6 of the $2.4 \mathrm{GHz}$ band, operating in $802.11 \mathrm{~g}$ mode.

In order to have a clean environment without any interference from other networks, both the LTE and the Wi-Fi equipment are placed in fully RF shielded boxes. These boxes are interconnected with each other using COAX cables through 
TABLE I. DL/UL TDD CONFIGURATIONS

\begin{tabular}{|c|c|c|c|c|c|c|c|c|c|c|c|}
\hline \multirow{2}{*}{$\begin{array}{l}\text { DL/UL } \\
\text { Config. }\end{array}$} & \multirow{2}{*}{$\begin{array}{l}\text { DL to UL } \\
\text { switch } \\
\text { periodicity } \\
\quad(\mathrm{ms})\end{array}$} & \multicolumn{10}{|c|}{ Subframe number } \\
\hline & & 0 & 1 & 2 & 3 & 4 & 5 & 6 & 7 & 8 & 9 \\
\hline 0 & 5 & D & $\mathrm{S}$ & $\mathrm{U}$ & $\mathrm{U}$ & U & $\mathrm{D}$ & $\mathrm{S}$ & U & U & $\mathrm{U}$ \\
\hline 1 & 5 & D & $\mathrm{S}$ & $\mathrm{U}$ & $\mathrm{U}$ & $\mathrm{D}$ & $\mathrm{D}$ & $\mathrm{S}$ & $\mathrm{U}$ & $\mathrm{U}$ & D \\
\hline 2 & 5 & D & $\mathrm{S}$ & $\mathrm{U}$ & D & $\mathrm{D}$ & $\mathrm{D}$ & $\mathrm{S}$ & U & D & D \\
\hline 3 & 10 & D & S & U & U & $\mathrm{U}$ & $\mathrm{D}$ & $\mathrm{D}$ & $\mathrm{D}$ & $\mathrm{D}$ & $\mathrm{D}$ \\
\hline 4 & 10 & D & $\mathrm{S}$ & U & U & $\mathrm{D}$ & D & $\mathrm{D}$ & D & D & D \\
\hline 5 & 10 & D & $\mathrm{S}$ & U & D & D & D & D & D & D & D \\
\hline 6 & 5 & D & $\mathrm{S}$ & $\mathrm{U}$ & $\mathrm{U}$ & $\mathrm{U}$ & $\mathrm{D}$ & $\mathrm{S}$ & $\mathrm{U}$ & $\mathrm{U}$ & $\mathrm{D}$ \\
\hline
\end{tabular}

TABLE II. SPECIAL SUBFRAME CONFIGURATIONS (OFDM SYMBOLS)

\begin{tabular}{|c|c|c|c|c|c|c|}
\hline \multirow{2}{*}{ Config. } & \multicolumn{3}{|c|}{ Normal CP } & \multicolumn{3}{c|}{ Extended CP } \\
\cline { 2 - 7 } & DwPTS & GP & UpPTS & DwPTS & GP & UpPTS \\
\hline 0 & 3 & 10 & 1 & 3 & 8 & 1 \\
\hline 1 & 9 & 4 & 1 & 8 & 3 & 1 \\
\hline 2 & 10 & 3 & 1 & 9 & 2 & 1 \\
\hline 3 & 11 & 2 & 1 & 10 & 1 & 1 \\
\hline 4 & 12 & 1 & 1 & 3 & 7 & 2 \\
\hline 5 & 3 & 9 & 2 & 8 & 2 & 2 \\
\hline 6 & 9 & 3 & 2 & 9 & 1 & 2 \\
\hline 7 & 10 & 2 & 2 & - & - & - \\
\hline 8 & 11 & 1 & 2 & - & - & - \\
\hline
\end{tabular}

combiners/splitters and programmable attenuators. Both the LTE and the Wi-Fi networks are configured in SISO (Single Input Single Output) mode. Hence, only one antenna port in both the transmitter and the receiver has been used.

\section{EXPERIMENTATION RESUlTS}

The purpose of this paper is to evaluate to which degree the performance of Wi-Fi is affected by a co-located LTE network transmitting in an overlapping frequency band in the way it was originally designed to operate, hence without any coexistence mechanism. Both the achieved Wi-Fi throughput and Wi-Fi round trip latency are adopted as key performance indicators. UDP traffic was sent for both the LTE and the Wi-Fi networks. The datagram size has been set to 1470 bytes and no RTS/CTS mechanism has been used by the Wi-Fi network.

When the Wi-Fi network does not experience any interference from LTE, the station is able to achieve an average DL throughput of $28.10 \mathrm{Mbps}$. In the remainder of the paper we refer to this throughput without LTE interference as $\mathrm{T}_{\text {REFF }}$. By monitoring the WLAN interface of the station, it has been noticed that in order to reach this throughput, the network used a high physical rate of 54Mbps. This is to be expected, as the RF shielded boxes and the interconnection via COAX cables offer an ideal, interference-free environment, where Wi-Fi can use a high Modulation and Coding Scheme (MCS) profile to transmit.

Fig. 3a presents an LTE frame of $10 \mathrm{~ms}$ in a time vs power measurement. This LTE frame includes only control signals in the DL, as there is no UE attached and consequently there is no traffic in the PDSCH nor in the PUSCH. This frame clearly shows the "3" TDD DL/UL configuration profile (TABLE I) that has been used, which consists of a DL subframe followed by a special subframe, 3 UL subframes and 5 DL subframes. As it can be observed, the control signals from the eNB are fairly sparse, offering many potential time slots to a co-located Wi-Fi network to transmit. This can be verified by Fig. 3b, which presents a Wi-Fi transmission alongside the eNB DL control signalling. It can be seen that Wi-Fi finds many opportunities to transmit covering the gaps between the LTE control signals. Fig. $3 \mathrm{c}$ depicts the time vs power signal measurement of simultaneous traffic by LTE and Wi-Fi with a duration of $10 \mathrm{~ms}$. This period of time equals to the duration of an LTE frame, in which LTE and Wi-Fi compete to access the medium. As can be seen, LTE sends DL or UL traffic during almost the whole time frame. Wi-Fi only has an opportunity to transmit during the special subframe, when LTE remains silent due to the guard period, between DL and UL transmissions.

One would expect that, since Wi-Fi has opportunities to transmit only during the GP, the throughput would be proportional to the duration of the GP, which depends on the special subframe configuration profile. Nonetheless, in the examined setup the LTE uses a $5 \mathrm{MHz}$ bandwidth, while the WiFi network operates in a $20 \mathrm{MHz}$ bandwidth. This means that LTE overlaps only with $25 \%$ of the Wi-Fi channel. Fig. 4 illustrates a $25 \mathrm{MHz}$ spectrum analysis during LTE and Wi-Fi transmissions. If the LTE signal is not strong enough to surpass the ED threshold of the CCA mechanism, or when LTE operates as hidden terminal, then Wi-Fi will not be able to sense the medium as busy and will attempt to transmit. This will cause LTE to interfere with Wi-Fi within the overlapping subcarriers. However, the OFDM modulation scheme that Wi-Fi uses in combination with the coding rate and error correction mechanisms render it capable to receive and decode data even though a part of the $20 \mathrm{MHz}$ spectrum is occupied. Despite the interference, a part of the transmitted packets can be successfully decoded at the receiver. Clearly, if the LTE bandwidth is higher, the amount of data that Wi-Fi would be able to decode will be lower. In case the detected LTE signal power is higher than the ED threshold, the Wi-Fi backoff mechanism is triggered.

In this study, we examine three different levels of LTE signal power in both the eNB and the UE. These power levels represent the different degrees that Wi-Fi can be affected by LTE operating in unlicensed spectrum in the way that it was originally designed.

The first one is symbolized as L1 and is not strong enough to surpass the ED threshold of the Wi-Fi CCA mechanism. Hence, Wi-Fi cannot sense the medium as busy and as a result LTE causes interference to ongoing Wi-Fi transmissions.

The second LTE power level is symbolized as L2. L2 is higher than the ED threshold of the Wi-Fi CCA and is able to force Wi-Fi to backoff every time there is an LTE transmission in the DL and in the UL.

The third examined LTE power level is symbolized as L3. At this level or above, LTE signals cause the surpassing of the sensitivity threshold at the Wi-Fi network. On modern Wi-Fi adapters the sensitivity threshold determines the lowest signal level for which the station remains associated with the current AP. If the signal level goes below this threshold the card disassociates and searches for a better AP.

TABLE III presents the different experimental scenarios that have been investigated and shows the measured Wi-Fi performance in terms of throughput and latency. Each scenario is defined by the type of LTE traffic together with the LTE signal power level. Fig. 5 summarizes the average measured values of Wi-Fi throughput and round trip latency for each scenario. 

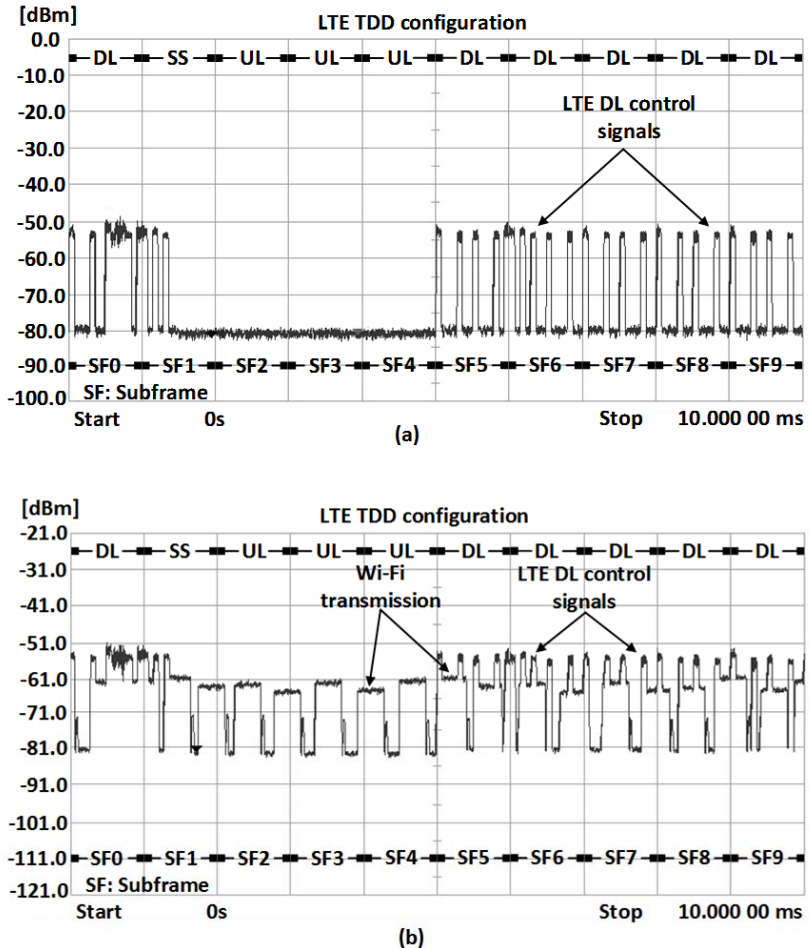

(b)

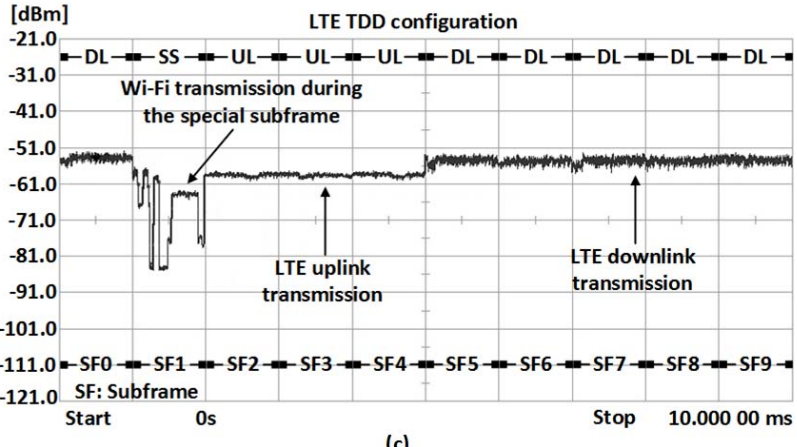

(c)

Fig. 3. Time vs. power analysis showing: a) LTE DL control signal transmissions b) Wi-Fi transmission opportunities between the LTE DL control signals c) Simultaneous LTE and Wi-Fi traffic.

The results show that even when LTE transmits only DL control signals, it is already able to cause severe interference to Wi-Fi, reducing its throughput drastically (Scenario 2 and 4). When LTE uses the L1 signal power, then its control signals interfere with the $\mathrm{Wi}-\mathrm{Fi}$ and decrease its throughput to $16.81 \mathrm{Mbps}$. This means that the Wi-Fi throughput has been reduced by $40.08 \%$ compared to $\mathrm{T}_{\mathrm{REF}}$. In the second case, when the L2 LTE signal power is used, the average Wi-Fi throughput reduction is even higher reaching $77.86 \%$ compared to $\mathrm{T}_{\mathrm{REF}}$.

When LTE transmits continuously in both the DL and UL, the throughput of Wi-Fi is decreased even more (Scenario 3 and 5). In the first case, in which LTE transmits using the L1 signal power, Wi-Fi does not sense the channel as busy. As Wi-Fi transmits concurrently with LTE an amount of useful information is lost due to the symbol mapping on the Wi-Fi subcarriers that face interference from LTE. If the receiver is not able to recover the lost information this will result to packet loss and retransmissions. As the experiment results show, the LTE

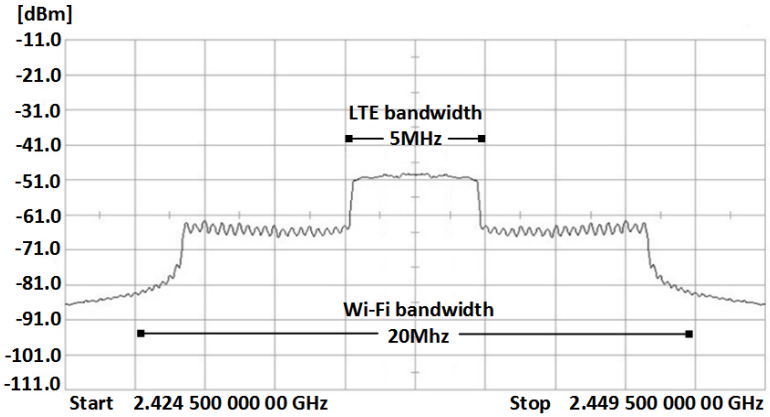

Fig. 4. The LTE and the Wi-Fi signal in frequency vs power analysis.

interference is strong enough to reduce the average $\mathrm{Wi}-\mathrm{Fi}$ throughput by $73.20 \%$ compared to $\mathrm{T}_{\mathrm{REF}}$. On the other hand, when LTE uses the L2 signal power, then Wi-Fi senses the medium as busy during DL and UL LTE transmissions. Thus, Wi-Fi is able to transmit only during the GP of the special subframe, in which LTE remains silent due to the switch between DL and UL. The results show that the average $\mathrm{Wi}-\mathrm{Fi}$ throughput in this case is limited to $1.84 \mathrm{Mbps}$ and equals to a degradation of $93.45 \%$.

By the time LTE starts transmitting using the L3 power level, it surpasses the sensitivity threshold of the Wi-Fi station. Hence, the Wi-Fi station disassociates from the AP and starts looking for another AP with better operating conditions (stronger signal, lower interference). In case there is no other AP that can serve the station, it remains disassociated. This way LTE completely eliminates Wi-Fi.

In terms of latency, the Wi-Fi network experiences an average round trip latency of $1.37 \mathrm{~ms}$, when there is no LTE activity (Scenario 1). By the time LTE is active the latency of Wi-Fi is significantly increased. When LTE uses the L1 signal power, then the presence of the LTE control signals in the DL raises the $\mathrm{Wi}-\mathrm{Fi}$ round trip latency to $1.6 \mathrm{~ms}$. This raise becomes even higher when LTE transmits continuously in both the DL and the UL and reaches the average value of $3.01 \mathrm{~ms}$. Furthermore, when the L2 signal power is used the LTE control signals in the DL increase the Wi-Fi latency by $117.5 \%$. The impact of concurrent DL and UL LTE traffic is even higher leading to an average latency increment by a factor of 3.32 .

\section{CONCLUSIONS AND FUTURE WORK}

This study has shown how the performance of a Wi-Fi transmission is affected by LTE using real hardware, when both technologies are co-located in unlicensed spectrum without any coexistence technique deployed. For the purpose of this study, we have used real LTE and Wi-Fi equipment in a fully

TABLE III. EXPERIMENTAL SCENARIOS

\begin{tabular}{|c|c|c|c|c|}
\hline $\begin{array}{c}\text { Scenario } \\
\text { ID }\end{array}$ & $\begin{array}{c}\text { LTE } \\
\text { signal } \\
\text { power } \\
\text { level }\end{array}$ & Type of LTE traffic & $\begin{array}{c}\text { Wi-Fi } \\
\text { throughput } \\
\text { value }\end{array}$ & $\begin{array}{c}\text { Wi-Fi latency } \\
\text { value }\end{array}$ \\
\hline 1 & - & none & $28.1 \mathrm{Mbps}$ & $1.37 \mathrm{~ms}$ \\
\hline 2 & L1 & DL CTRL signal & $16.81 \mathrm{Mbps}$ & $1.6 \mathrm{~ms}$ \\
\hline 3 & L1 & DL and UL traffic & $7.53 \mathrm{Mbps}$ & $3.01 \mathrm{~ms}$ \\
\hline 4 & L2 & DL CTRL signal & $6.22 \mathrm{Mbps}$ & $2.98 \mathrm{~ms}$ \\
\hline 5 & L2 & DL and UL traffic & $1.84 \mathrm{Mbps}$ & $5.92 \mathrm{~ms}$ \\
\hline 6 & L3 & DL CTRL signal & disassociated & disassociated \\
\hline 7 & L3 & DL and UL traffic & disassociated & disassociated \\
\hline
\end{tabular}




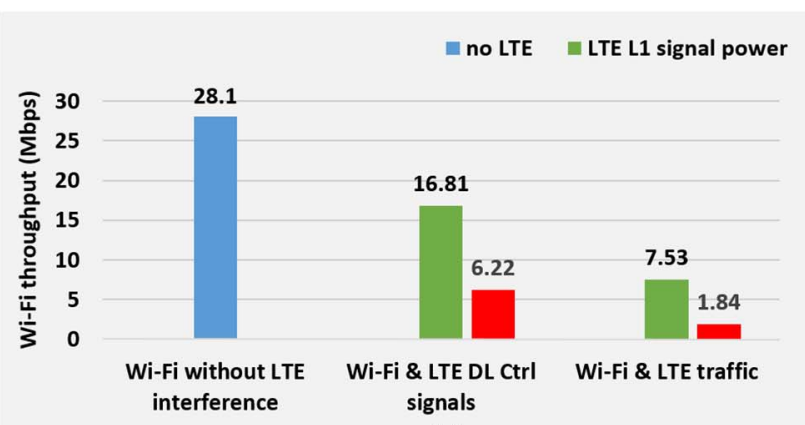

(a)

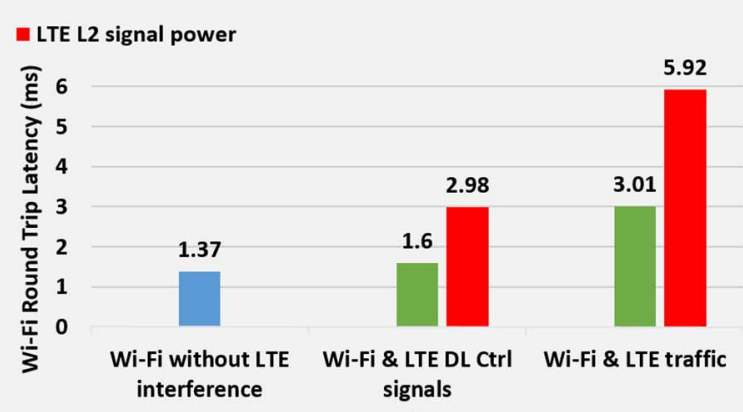

(b)

Fig. 5. Wi-Fi average throughput and round trip latency in standalone operation and under different levels of LTE interference.

controlled wireless environment. The results show that the WiFi performance is severely affected by LTE in terms of achieved throughput and latency. We show that even if LTE does not send data traffic, the throughput of Wi-Fi is reduced significantly due to the LTE control signals. Furthermore, this reduction becomes even more pronounced when LTE transmits arbitrarily in both PDSCH and PUSCH channels. Three different levels of LTE signal have been examined, each one representing the different level of impact that LTE may have on Wi-Fi. As the results showed even if the LTE signalling does not surpass the ED threshold of $\mathrm{CCA}$, the Wi-Fi transmissions experience significant interference from LTE. In case the LTE signal is higher than the ED threshold, it forces Wi-Fi to backoff and under concurrent DL and UL transmission it gives opportunities to the medium only during the GP period of the TDD configuration. Finally, when the LTE transmission power exceeds the Wi-Fi sensitivity threshold, it forces the Wi-Fi station to disassociate from the AP, eliminating this way the WiFi network. If there are many UEs in the LTE network, the resources would be divided to the UEs by the LTE scheduler. In a heavy loaded network, the impact on Wi-Fi is expected to be at least the same as the examined case in this paper, where there is continuously DL traffic to one UE. In case there are multiple Wi-Fi STA, then the impact on Wi-Fi is expected to be higher as more STA would compete during the idle slots. The verification of these assumptions has been left for future work.

From the results above, it is clear that the design and implementation of coexistence mechanisms are needed in order to achieve a harmonized coexistence between LTE and Wi-Fi in the unlicensed spectrum. The main reason that LTE interferes with Wi-Fi is because it does not sense the medium before a transmission. By enhancing LTE with a carrier sensing mechanism it would be able to avoid interference with other ongoing transmissions and backoff or move to another channel using a DFS (Dynamic Frequency Selection) technique. 3GPP has already started working on the definition of standards towards the enhancement of LTE with CCA. Another dominant solution is the scheduling of blank subframes in LTE. This solution is applicable to regions where no CCA requirements are defined. During these subframes LTE would remain silent, giving Wi-Fi the opportunity to transmit. In this case, we could consider similar techniques used in enhanced Inter-Cell Interference Coordination (eICIC), where LTE subframes in a certain cell are reserved for neighbouring cells. This solution requires a careful and sophisticated selection of the amount of blank subframes, in order to keep a balance between sufficient Wi-Fi transmission opportunities and LTE performance. In the near future, we are planning to further contribute to the ongoing research and standardization towards the compelling coexistence between LTE and Wi-Fi and propose potential improvements.

\section{ACKNOWLEDGMENT}

The research leading to these results has received funding from the European FP7 Program under grant agreement FP7612050 (FLEX project).

\section{REFERENCES}

[1] Qualcomm, "Rising to meet the 1000x mobile data challenge", 2012

[2] 3GPP news, "Evolution of LTE in release 13", February 2015, http://www.3gpp.org/news-events/3gpp-news/1628-rel13

[3] “LTE-U Forum", http://www.lteuforum.org/

[4] "MulteFire Alliance", http://www.multefire.org/

[5] J. Liu, Y. Kawamoto, H. Nishiyama, N. Kato and N. Kadowaki, "Deviceto-device communications achieve efficient load balancing in LTEadvanced networks", IEEE Communications Society, pp. 57-65, April 2014

[6] Huawei, "U-LTE: Unlicensed spectrum utilization of LTE”, 2014

[7] R. Ratasuk, et.al., "License-exempt LTE deployment in heterogeneous network", IEEE, Paris, pp. 246-250, Augustus 2012

[8] T. Nihtila, et.al., "System performance of LTE and IEEE 802.11 coexisting on a shared frequency band", IEEE, Shanghai, pp. 1038-1043, April 2013

[9] E. Almeida, et.al., "Enabling LTE/WiFi coexistence by LTE blank subframe allocation", Budapest, pp. 5083-5088, June 2013

[10] F. Liu, E. Bala, E. Erkip and R. Yang, "A framework for femtocells to access both licensed and unlicensed bands", IEEE, Princeton, NJ, pp. 407411, May 2011

[11] S. Sagari, et.al., "Coordinated dynamic spectrum management of LTE-U and Wi-Fi Networks", IEEE DySPAN, pp/ 209-220, Sept.-Oct. 2015

[12] IEEE standard 802.11, "Wireless LAN medium access control (MAC) and physical layer (PHY) specifications, 2012."

[13] 3GPP TS 36.211, Evolved Universal Terrestrial Radio Access (EUTRA), "Physical Channels and Modulation", (Release 12).

[14] "European FP7 FLEX project portal", http://www.flex-project.eu

[15] "iLab.t iMinds testbed", http://doc.ilabt.iminds.be/ilabt-documentation/

[16] "ExpressMIMO2 board specifications", http://openairinterface.eurecom.fr/expressmimo2

[17] N. Nikaein, et.al., "Openairinterface: A flexible platform for $5 \mathrm{~g}$ research", ACM SIGCOMM Computer Communication Review, volume 44, issue 5, pp. 33-38, October 2014

[18] "ath9k wireless driver Atheros IEEE 802.11n PCI/PCI-Express and AHB WLAN based chipsets", http://linuxwireless.org/en/users/Drivers/ath9k/ 